\title{
IMPORTANCIA DE INTEGRAR LA CALIDAD A LA CULTURA ORGANIZACIONAL EN INSTITUCIONES DE EDUCACIÓN SUPERIOR
}

\section{IMPORTANCE OF INTEGRATING QUALITY TO ORGANIZATIONAL CULTURE IN INSTITUTIONS OF HIGHER EDUCATION}

\begin{abstract}
Mónica del Pilar Llanos Encalada, Ph.D. Doctora en Ciencias Económicas (Cuba). Docente Titular de la Facultad de Ciencias Económicas y Empresariales de la Universidad Tecnológica ECOTEC, Ecuador. mllanos@ecotec.edu.ec
\end{abstract}

\section{ARTÍCULO DE REFLEXIÓN}

Recibido: 18 de octubre de 2019.

Aceptado: 28 de noviembre de 2019.

\section{RESUMEN}

Las constantes exigencias del entorno, hace que las organizaciones de educación superior estén en permanente innovación y búsqueda de la calidad, ya no solo los organismos de control exigen calidad, también los estudiantes y comunidad demandan una formación profesional de calidad. Las instituciones educativas buscan que la calidad sea permanente y no solo para periodos de evaluación. La cultura organizacional es la personalidad de la organización y constituye los patrones de principios y hábitos de comportamiento, por lo cual se ve necesario que esta cultura esté impregnada de calidad, ya que esta permite integrar las características de las personas a las características de las organizaciones. Varios son los organismos en diferentes regiones que han aportado a que la calidad forme parte del accionar permanente en cada uno de los procesos sustantivos de la educación superior. En 14 estudios de la literatura consultada se evidencia que para lograr desarrollar la calidad es necesario integrarla como parte de la cultura de la organización y, por lo tanto, de cada uno de sus miembros, para que exista un apropiamiento en cada accionar al interior 
de las organizaciones, será necesario que la institución cree un modelo de cultura organizacional de manera consciente.

Palabras clave: educación superior, cultura organizacional, calidad.

\section{ABSTRACT}

The constant demands of the environment, makes higher education organizations in constant innovation and pursuit of quality, and not only control bodies demand quality, also students and community demand quality professional training. The educational institutions seek that the quality be permanent and not only for evaluation periods. The organizational culture is the personality of the organization and constitutes the patterns of behavior principles and habits, so it is necessary that this culture be impregnated with quality, since this allows integrating the characteristics of people to the characteristics of organizations. There are several organizations in different regions that have contributed to quality being part of the permanent action in each of the substantive processes of higher education. In 14 studies of the literature consulted, it is evident that in order to develop quality it is necessary to integrate it as part of the culture of the organization and therefore of each of its members, so that there is an appropriation in each action within organizations, it will be necessary for the institution to create a model of organizational culture in a conscious way.

Keywords: higher education, organizational culture, quality.

\section{INTRODUCCIÓN}

Los principios de administración y gestión empresarial han sido acogidos por instituciones de educación superior. Al ser parte del mundo globalizado, no pueden estar ajenas al avance de la ciencia, tecnología, y conocimiento. Deben estar a la vanguardia de los retos de la "Era del conocimiento y la comunicación", más aún cuando forman profesionales que se van a desempeñar en posiciones claves en el mundo empresarial, político, económico y social de los países.

ISSN: 1390-9320, Edición Especial, febrero 2020 
Hasta las primeras décadas del siglo XX, las universidades eran consideradas claustros del saber, aisladas del entorno y la realidad circundante, con acceso limitado a grupos privilegiados. Luego se da paso la masificación de la educación y el libre acceso, lo cual lleva a que las universidades públicas no se den abasto a la creciente demanda. Surgen instituciones particulares, en algunos casos esto trajo consigo la proliferación indiscriminada y el interés mercantilista de algunas personas, afectando la calidad en la educación. A criterio de la autora, "se dio paso a la masificación de la educación con libre acceso, pero en detrimento de la calidad".

En el caso de Ecuador, las universidades se ven obligadas a cumplir requerimientos con carácter legal, tales como: estudios de cuarto nivel de sus profesores, docentes investigadores, publicaciones científicas, vinculación con problemas reales de la sociedad y otros, que no han sido desconocidos en su mayoría, pero que fueron postergados por no ser considerados prioritarios. El no cumplimiento se debe en gran parte a la falta de preparación investigativa, pero especialmente por no contar con una cultura institucional que promueva todos estos cambios (Llanos, 2014). Frente a este escenario, las universidades no se encontraban en igualdad de condiciones frente a las exigencias establecidas por el Consejo de Evaluación y Aseguramiento de la Calidad en la Educación Superior (Ceaaces).

Existen tres tipos de instituciones: las públicas, las privadas cofinanciadas y las privadas autofinanciadas. En la primera evaluación del 2009, se evaluaron las 68 universidades existentes. Para la segunda fase de evaluación, cuyo informe se emitió en el 2013, la categoría "E", fue eliminada, aquellas que no habían logrado cumplir con el mínimo de calidad exigida, salieron del sistema educativo (14 universidades). Todas las universidades evaluadas en la segunda fase, debían contar con la aspiración y planes de mejora que las lleve a ocupar las categorías "A" y "B".

Este escenario lleva a la búsqueda de alternativas que permitan a las universidades cumplir con estándares e indicadores de calidad, por ello el objetivo del presente estudio es reflexionar sobre la importancia de integrar la calidad como parte de la cultura 
organizacional como estrategia para que la primera se instaure como parte intrínseca de las personas de las instituciones de educación superior.

\section{REVISIÓN TEÓRICA}

\section{La búsqueda de la calidad en instituciones de educación superior.}

En la primera década del siglo XXI, surge la preocupación de organismos internacionales de primer mundo de impulsar el desarrollo de América Latina y el Caribe, mediante el logro de la calidad y estandarización en la formación recibida por los profesionales; más aún cuando el mundo demanda de ellos una formación integral, sólida, preparados para enfrentar los retos de su comunidad local, regional, nacional e internacional.

Surgen organismos como el Instituto Internacional para la Educación Superior de América Latina y el Caribe (IESALC), organismo adscrito a la Organización de Naciones Unidas para la Educación, la Ciencia y la Cultura (UNESCO); La Red Iberoamericana para la Acreditación de la Calidad en la Educación Superior (RIACES); El Consejo Nacional de Evaluación y Acreditación de la Educación Superior del Ecuador (CONEA); Agencia Nacional de Evaluación y Acreditación de Educación Superior de Paraguay (Aneaes); Comisión Nacional de Evaluación y Acreditación Universitaria de Argentina (CONEAU); Consejo Nacional de Acreditación Colombia (CNA- Colombia). (CONEA, 2003)

En la conferencia regional de la UNESCO, realizada en la Habana (1996), sobre Calidad de la Educación Superior, se plantea que "la calidad puede definirse como la adecuación del ser y del quehacer de la educación superior, a su deber ser" (CONEA, 2003). El "ser" para la autora es la cultura como la personalidad que da identidad a las organizaciones, por lo tanto, si no se trabaja en el desarrollo de un modelo de cultura organizacional que integre la calidad y sus componentes, el cumplimiento se verá reflejado en la presión de último momento por cumplir lo que no se ha gestado en el tiempo.

ISSN: 1390-9320, Edición Especial, febrero 2020 
Por ello, la calidad en la educación superior es multidimensional, incluye características universales y particulares que aluden a la naturaleza de las instituciones y los problemas que se plantean en los distintos contextos sociales en el marco de prioridades nacionales, regionales y locales (CONEA, 2003).

EI IESALC, plantea puntos claves para lograr la calidad en la Educación Superior en América Latina y el Caribe: integración regional; movilidad académica-profesional; ofertas transnacionales y acuerdos de beneficio mutuo y para lograrlo, se necesita enfrentar los siguientes desafíos:

a) Expansión de la educación con calidad y equidad;

b) Vinculación de la universidad con la comunidad a través de proyectos nacionales y regionales;

c) Formación de una conciencia social que reconozca la diversidad y a la vez valorice la identidad cultural;

d) Capacitación al recurso humano para que sea capaz de competir;

e) Armonización de los sistemas educativos;

f) Cooperación interuniversitaria para la optimización de recursos, intercambio de experiencias en especial en investigación;

g) Creación y fortalecimiento de sistemas de evaluación, acreditación como instrumentos para garantizar la calidad;

h) Formulación e implementación de políticas públicas pertinentes.

i) Creación de una cultura de innovación para la estrecha cooperación entre el sector industrial y empresarial.

j) Comprensión del sistema de Educación Superior como instrumento estratégico para el desarrollo sustentable e inserción competitiva de los países de América Latina y el Caribe en la sociedad del conocimiento (IESALC, 2007).

Entre los desafíos mencionados, hay aspectos relacionados a la cultura: conciencia social, identidad cultural, talento humano competitivo y creación de una cultura de innovación; por lo cual, la base fundamental será diagnosticar la cultura de cada organización para 
implementar estrategias que permita que los componentes de calidad formen parte de los valores, principios y convicciones que caracteriza a los miembros que conforman las instituciones y luego que estos se vean reflejados en las prácticas diarias de la comunicación, el liderazgo, las relaciones y estilos de trabajo.

Otro aporte en esta misma línea es el Proyecto Tuning, financiado por la Comisión Europea en el marco del programa Sócrates, creado para responder al reto de la Declaración de Boloña y del Comunicado de Praga. El propósito de este proyecto fue contribuir a la educación mediante acuerdos que propendan a mejorar la calidad. Bajo este esquema se adopta el modelo para los países latinoamericanos, mediante el Proyecto Alfa Tuning América Latina. La primera fase del Proyecto Tuning (2004 - 2008), y la segunda fase (2011 - 2013). En esta última se integra Ecuador.

Sobre rankings y mediciones científicas se comenta mucho en los últimos años en el mundo hispano: la competitividad y la producción científica es uno de los índices de desarrollo y de posibilidades de crecimiento económico y social...Por otra parte, estar en el top de los rankings significa tener mayores posibilidades de financiación pública y privada, y mayor demanda en el mercado global, lo que es esencial para las propias instituciones investigadoras (Turning América Latina, 2012).

Al momento de determinar la calidad en la educación superior, aparte de indicadores establecidos por cada nación, se deben considerar aquellos de orden regional y mundial, ya que la educación es considerada una estrategia del desarrollo social y económico de los países. "Para avanzar en la senda de la sociedad del conocimiento, los países de América Latina y el Caribe deberán masificar aún más sus sistemas de educación terciaria, robustecer su capacidad científica y tecnológica propia, y conectarse mejor con su sociedad" (UNESCO, 2013).

Entre los indicadores de calidad, constan: investigaciones, publicaciones y propiedad intelectual. Los países de América Latina que llevan la delantera en estos indicadores son: Chile, Uruguay, Argentina, Brasil y México. En lo que se refiere a publicaciones científicas, 
Ecuador ocupa el lugar 13 de los países de América Latina y el quinto en patentes (IESALC, 2007).

El Ministerio de Educación Superior (MES) de la República de Cuba desde su creación (1976), da importancia al control del trabajo en instituciones de educación superior mediante procesos de evaluación para un mejoramiento continuo de calidad (Horruitiner, 2007). Los ámbitos de calidad en este sistema se asocian a las personas (docentes y estudiantes); material disponible (equipamiento y tecnología), y el proceso de formación (metodología y pertinencia).

La calidad en instituciones de educación superior, no solo constituye cumplimiento frente a organismos de control, también la percepción de usuarios de los servicios universitarios. "La percepción de la calidad del servicio por parte del estudiante es de gran interés, porque en la actualidad existen más universidades tanto públicas como privadas que compiten por estar certificadas..." (Reyes \& Reyes, 2012).

Es sorprendente que esta tendencia que lleva algunos años promoviéndose y que constaba en la ley de educación superior del Ecuador del 2000, no haya incidido oportunamente en el cambio de una cultura al interior de las instituciones. Fueron pocos los avances en las instituciones de educación superior según los informes de evaluación presentados por el CONEA.

Las universidades, previo a los procesos de evaluación, ya contaban con un proyecto de autoevaluación institucional, se socializaron los estándares e indicadores a través de varios documentos, los cuales no difieren mucho con los actuales. Sin embargo, muchas instituciones fueron sorprendidas, quizás porque se consideraba que se podía evadir y dilatar en forma continua e interminable el cumplimiento de una gestión de calidad, como se había hecho costumbre en tantos sectores de Ecuador. La educación no sería la excepción. 
La Asamblea Nacional Constituyente (ANC), dictamina el Mandato Constituyente No. 14 el 22 de julio del 2008, en el cual solicita al CONEA, que emita un informe al proceso de evaluación iniciada, en la cual se consideraron los siguientes criterios: infraestructura, gestión, currículo. Es así que, en el año 2009 se da a conocer que, de las 68 universidades existentes en el país, solo 11 cumplían los estándares de calidad; lo cual da lugar a la categorización de universidades, clasificadas de la siguiente manera: 9 en categoría "B", 13 en "C", 9 en "D” y 26 en "E”.

Luego, en la disposición transitoria de la Ley de Educación Superior (LOES) del 12 de octubre del 2010, se dispone al Consejo de Evaluación, Acreditación y Aseguramiento de la Calidad (Ceaaces)- conformado para reemplazar al CONEA- que, en los 18 meses posteriores a ser emitida la ley, se proceda a evaluar a las universidades que obtuvieron la categoría "E". Dicho proceso se dio a inicios del 2012, considerando los mismos criterios de evaluación anterior, además la evaluación académica a estudiantes. Los resultados de esta evaluación a las 26 instituciones, 3 alcanzaron niveles aceptables, 9 parcialmente aceptables y 14 fueron cerradas (Ceaaces 2012).

En el año 2013 se da paso a la segunda fase de evaluación a las universidades y escuelas politécnicas por parte del Ceaaces, previamente se dieron mesas de trabajo con representantes de algunas instituciones, para la socialización e intercambio de opiniones en el establecimiento de los nuevos criterios de evaluación: Academia, Eficiencia Académica, Investigación, Organización e Infraestructura. Los resultados una vez más sorprendieron a las instituciones de educación superior al darse una reclasificación. En unos casos algunas instituciones subieron de categoría y en otros casos bajaron (Ceaaces 2013). Muchas inconformidades se han dado; sin embargo, es importante hacerse una serie de cuestionamientos al interior de las instituciones educativas y tomar acciones que permitan alinearse a los requerimientos exigidos.

Es de conocimiento general los avances en la calidad de la educación superior pública y privada en Ecuador posterior a los procesos de evaluación. El Art. 173 de la Ley Orgánica de Educación Superior (LOES), determina que: "Ceaaces, normará la autoevaluación 
institucional, y ejecutará los procesos de evaluación externa, acreditación, clasificación académica y el aseguramiento de la calidad" (Asamblea Nacional del Ecuador, 2010). Se enfatiza; además, que el cambio de imaginarios colectivos se debe realizar a través de programas de comunicación y educación con la finalidad de que los cambios sean significativos en la época que vive el país. Para la autora, los "imaginarios colectivos" están referidos a la cultura organizacional, puesto que si las nuevas tendencias y exigencias en calidad no se integran como parte de la cultura individual e institucional es poco lo que se puede lograr.

En algunos lineamientos de las políticas de gobierno del Plan Nacional de Desarrollo del Buen Vivir (PNBV 2013-2017), se menciona la creación de una Cultura de paz, emprendimiento y excelencia, además una cultura de calidad y calidez en cada sector del país, entre ellos la educación. Se enfatiza que los principios establecidos deben formar parte de la cultura organizacional. En el PNBV, la política 2.5 menciona: "Fomentar la inclusión y cohesión social, la convivencia pacífica y la cultura de paz, erradicando toda forma de discriminación y violencia", por lo tanto, la calidad es considerada parte de la cultura (SENPLADES, 2013).

\section{Importancia de la Cultura Organizacional para el desarrollo de la calidad.}

Existen varios estudios sobre cultura organizacional y calidad en instituciones de educación superior, de los cuales, la autora ha seleccionado 14 investigaciones: Kauffman (1997) realiza el estudio sobre la cultura de calidad total para la Universidad de Veracruz, a partir de los modelos de calidad conocidos en el ámbito empresarial, realiza una confrontación del diagnóstico obtenido. Ismael (2003), estudia la cultura organizacional y la cultura de calidad en la educación superior, relacionando estas variables con el clima y la calidad del desempeño docente. Arce (2005), analiza la cultura universitaria basada en la calidad y la excelencia. Luego, Piva (2006), realiza el análisis de la cultura en una de las facultades de Argentina. 
Elba Barciela (2007), realiza su estudio en las sedes universitarias municipales (SUM) en Cuba, relacionando la cultura con el comportamiento, a partir de una metodología que la caracteriza. En esta postura, la cultura adopta un enfoque funcionalista al permitir el desempeño de la organización con respecto de la racionalidad económica y la visión desde el desarrollo organizacional. El otro enfoque se refiere a que la organización es cultura, metáfora que sirve para expresar la connotación socio-antropológica de la organización. "La cultura se hace metáfora y la organización es cultura, es la organización de la organización, entorno, sentido y razón, logros compartidos trasmitidos por el lenguaje, la imagen y los símbolos" (Barciela, 2007).

(Botello, 2008) diseña un modelo en el cual integra las dimensiones de cultura con principios de calidad para la formación del bachiller, para lo cual propone estrategias para su implementación. Existen dos modelos de análisis de cultura organizativa en universidades públicas catalanas, el primero a nivel macro de la institución y el segundo a nivel micro de una facultad. Los denominados modelos se remiten a la elaboración de cuestionarios para cada caso (Thomás \& Ion, 2008).

Marina Tomás; Nuria Borrell; Diego Castro (2009), realizan una investigación para implementar una "cultura innovadora" para universidades de España. Luego, Marina Tomás y David Rodríguez (2009), diseñan un modelo de evaluación de la cultura a partir de diez dimensiones, para universidades españolas. Hernández (2012), realiza un estudio sobre calidad y excelencia vinculados a la cultura (valores) en instituciones de educación superior.

Se plantea este dilema porque en la actualidad la universidad está destinada a cambiar si quiere continuar siendo la institución que cree, critique y transmita conocimiento para el desarrollo de la sociedad. Debe, por lo tanto, dotarse de una cultura organizativa predispuesta al cambio. Sin embargo, para poder llegar a adoptar una cultura de cambio, es necesario que adopte estrategias que contribuyan a modificar la cultura existente para convertirla en una cultura en la que los valores y conductas predominantes sean las de aceptación al cambio, interés para mejorar, etc. características de una cultura innovadora. (Tomás, y otros, 2009).

ISSN: 1390-9320, Edición Especial, febrero 2020 
En la Universidad Central "Martha Abreu" de las Villas, (González, Fernández, \& Hernández, 2013), proponen un procedimiento para el diagnóstico de "una cultura de calidad", considerada como estrategia de gestión por el Ministerio de Educación Cubano (2010). Para su estudio parten de dos componentes: el psicológico referido a valores, creencias, expectativas y compromiso a la calidad, y el estructural administrativo caracterizado por procesos definidos y coordinación de esfuerzos individuales.

Pujol, Foutel, \& Musticchio (2014) realizan un estudio preliminar que aborda la cultura en relación a la identidad y clima organizacional. Posteriormente, García, Muratalla, \& Gaona (s.f.), estudian la cultura y la calidad, considerando la cultura como eje estratégico para instituciones de educación superior que cuenten con sistemas de gestión de la calidad. Vesga (2013) considera en su estudio la premisa de que la cultura organizacional y los sistemas de gestión de calidad constituyen una relación clave para la gestión de las instituciones de educación superior.

Como se aprecia en la tabla 1, en las investigaciones realizadas por los autores referenciados, todos dan importancia trascendental a aspectos vinculados a la gestión interna en instituciones de educación superior, ocho de estos vinculan directamente a la calidad con la cultura organizacional mientras que tres proponen procedimientos para evaluar la cultura y los otros tres vinculan la cultura con la identidad, clima laboral, comportamiento.

Tabla 1. Síntesis de los aportes de investigaciones realizadas.

\begin{tabular}{ll}
\hline \multicolumn{1}{c}{ Autores } & \multicolumn{1}{c}{ Estudio Central } \\
\hline Kauffman (1997) & Calidad Total a partir de modelos de \\
& calidad del ámbito empresarial. \\
Ismael (2003) & Cultura organizacional y cultura de \\
& calidad relacionadas al clima y calidad del \\
Arce (2005) & desempeño. \\
& La cultura universitaria basada en la \\
& calidad y la excelencia. \\
\hline
\end{tabular}

ISSN: 1390-9320, Edición Especial, febrero 2020 


\begin{tabular}{|c|c|}
\hline Piva (2006) & Análisis de la cultura. \\
\hline Barciela (2007) & $\begin{array}{l}\text { La cultura con el comportamiento } \\
\text { organizacional. }\end{array}$ \\
\hline Botello (2008) & $\begin{array}{l}\text { Integra las dimensiones de cultura con } \\
\text { principios de calidad. }\end{array}$ \\
\hline Thomás \& Ion (2008) & Modelos de evaluación de la cultura \\
\hline $\begin{array}{l}\text { Marina Tomás; Nuria Borrell; Diego } \\
\text { Castro (2009) }\end{array}$ & Cultura Innovadora. \\
\hline Marina Tomás y David Rodríguez (2009) & $\begin{array}{l}\text { Evaluación de la cultura a partir de diez } \\
\text { dimensiones. }\end{array}$ \\
\hline Hernández C. (2012) & $\begin{array}{l}\text { Calidad y Excelencia vinculados a la } \\
\text { cultura. }\end{array}$ \\
\hline $\begin{array}{l}\text { González, Fernández, \& Hernández } \\
\text { (2013) }\end{array}$ & $\begin{array}{l}\text { Procedimiento para el diagnóstico de la } \\
\text { cultura. }\end{array}$ \\
\hline Pujol, Foutel, \& Musticchio (2014) & $\begin{array}{l}\text { La cultura en relación a la identidad y } \\
\text { clima organizacional. }\end{array}$ \\
\hline García, Muratalla, \& Gaona (s.f.) & $\begin{array}{l}\text { La cultura y la calidad. La primera como } \\
\text { eje estratégico. }\end{array}$ \\
\hline Vesga (2013) & $\begin{array}{l}\text { La cultura organizacional y sistemas de } \\
\text { gestión de calidad, relación clave para la } \\
\text { gestión en instituciones de educación } \\
\text { superior. }\end{array}$ \\
\hline
\end{tabular}

Fuente: Elaboración propia a partir de aportes de autores consultados en la literatura.

La autora considera que es importante que las universidades ecuatorianas se interesen en hacer un diagnóstico de la cultura de sus organizaciones, lo cual les va a permitir plantear el modelo de cultura que se quiere implementar, acorde a las características particulares y lineamientos generales, entre los cuales la calidad debe ser parte de sus dimensiones para que estén integradas en cada uno de los miembros de la comunidad universitaria. 


\section{CONCLUSIONES}

Las universidades han iniciado un proceso de desarrollo y mejoramiento continuo que empezó a inicios del siglo XX y se incrementa en lo que va del siglo actual, por lo cual la Calidad constituye un elemento intrínseco a su gestión e identidad institucional.

El aporte y las iniciativas realizadas por varios de los organismos internacionales han logrado su eco y el fruto en el proceso irreversible y progresivo de asegurar la calidad en las instituciones de educación superior de América Latina, evidenciado en Ecuador.

Los avances logrados en las instituciones de educación superior ecuatorianas, se han visibilizado en los procesos de evaluación por parte de organismos de control.

Aún la calidad, evaluación y mejora permanente debe institucionalizarse y para ello, la cultura constituye el aliado fundamental para lograrlo.

Las universidades deben desarrollar una cultura organizacional que integre los principios, estándares e indicadores de calidad, de tal forma que sean apropiados, integrados y practicados por todos sus miembros en cada una de las tareas desempeñadas.

\section{REFERENCIAS BIBLIOGRÁFICAS}

Arce, C. (febrero de 2005). La Cultura Universitaria basada en la calidad y la excelencia. San José, Costa Rica.

Asamblea Nacional del Ecuador. (12 de octubre de 2010). Ley Orgánica de Educación Superior. Quito, Ecuador: Lexus.

Barciela, E. (2007). Bases teórico- metodológicas para un enfoque de la gestión de la cultura orgnizacional en las sedes universitarias municipales de Cuba. ACIMED: Volúmen 16, No 2, 15.

ISSN: 1390-9320, Edición Especial, febrero 2020 
Bobadilla, M., Callata, C., \& Caro, A. (2015). Compromiso laboral y cultura organizacional, el rol de la orientaciòn cultural en una empresa. Tesis para la optenciòn del grado científico de Magister en Desarrollo Organizacional y Direcciòn de empresas. Universidad del Pacifico.

Botello, M. (Julio de 2008). Modelo de Cultura de Calidad para la direcciòn de formación del bachiller. Ciencia UANL, 11(3), 233-243.

Camarena, M. E., \& Rodríguez, A. (2010). Dimensiones de la Cultura Organizacional: Bases para su implementación. Monterrey: XIV Congreso Internacional de la Academia de Ciencias Administrativas A. C. (ACACIA).

Ceballos, K., \& Ceballos, G. (2008). Diagnóstico del Desarrollo Organizacional- 2006 en una empresa productora de aceite de Palma Africana, en Santa MarthaColombia. Clío -América- Universidad de Magdalena, 111-121.

Felcman, I., Blutman, G., \& Méndez, M. S. (2001). Modelos de Cultura Organizacional en la Administración Pública Argentina. Centro de Investigaciónes en la Administración Pública Argentina.

Figueroa, L. (2015). Cultura Organizacional y Desempeño en una organización gubernamental. Tesis en optenciòn al título de licenciado en Psicologìa Industrial Organizacional. Guatemala: Universidad Rafael Landívar.

Freire, M., Talamini, E., \& De Oliveira, L. (s.f.). Diagnóstico de Clima Organizacional utilizando la matriz de importancia del desempeño, aplicado en una pequeña empresa de agronegocio. Micro o Pequeña Empresa.

García, J., Muratalla, G., \& Gaona, H. (s.f.). Cultura Organizacional, eje estratégico en las instituciones de educación superior con sistemas de gestión de la calidad. XXI Congreso Internacional de Contaduría, Admiistracion e Informática. México: Asociación Nacional de facultades de Contaduría, Administración e Informática.

González, A., \& Fernández, E. (2000). La Cultura de la Organización en la Gestión de la Calidad Total. Ensaios e Ciencia, 99-114.

ISSN: 1390-9320, Edición Especial, febrero 2020 
González, E., Fernández, M., \& Hernández, G. (julio a diciembre de 2013). Diagnóstico de la Cultura de la Calidad en una facultad universitaria. Estrategia y Gestión Universitaria, 1(1).

Hernández, C. (2012). La calidad y la excelencia ¿un problema de cultura y valores en las organizaciones? Ciencia Administrativa, 92-101.

IESALC (2007). Congreso Internacional: La Acreditación de la Calidad de la Educación Superior de Cara al Siglo XXI. La Acreditación de la Educación Superior para la generación de profesionales del siglo XXI (pág. 14). QuitoEcuador: CONEA.

Ismael, U. (2003). Aplicación de Cultura Organizacional y Cultura de Calidad. México: Universidad Autónoma Nueva León.

Kauffman, S. (1997). Una Cultura de Calidad para la Universidad Veracruzana. Ciencias Administrativas, 5-18.

Llanos, M. (2014). La Cultura Universitaria para el desarrollo en la ciencia y tecnología en respuesta a las demandas sociales en Latinoamérica. Congreso Internacional "Tecnología, Universidad y Sociedad" p. 12. Santo DomingoRepública Dominicana: Universidad Central del Este.

Llanos, M. (2014). La Educación Superior en Interacción con la Sociedad Actual. (C. d. Conocimiento, Ed.) Estrategia y Gestión Universitaria, Vol. 2, No. 1.

Llanos, M. (2015). La Cultura: Estrategia en la Calidad del Servicio al Cliente. Madrid: Editorial Académica Española.

Ospina, D. (2014). ¿Por qué un modelo de Cultura Organizacional? Tesis para la opción al título de especialidad en Gestión de Desarrollo Administrativo. Colombia: Universidad Militar Nueva Granada.

Piva, A. M. (2006). Cultura Organizacional: Un caso de análisis. Título para la optención de grado de licenciado en Sociología. Argentina: Facultad de Humanidades y Ciencias de la Educación.

ISSN: 1390-9320, Edición Especial, febrero 2020 
Pujol, L., Foutel, M., \& Musticchio, A. (2014). Hacia la Construcciòn de un modelo para el abordaje empírico de la identidad y la cultrua organizacional: Resultados preliminares. Argentina: Universidad Nacional del Mar del Plata.

RAE. (05 de mayo de 2017). Real Academia de la Lengua Española. Recuperado de dle.rae.es/¿id=PTk5Wk1

Reyes, O., \& Reyes, M. (2012). Percepción de la calidad del servicio de la Educación Universitaria de alumnos y profesores. Revista Internacional Administración y Finanzas, 5(5). Recuperado de ftp.repec.org > RePEc > ibf > riafin > riaf-v5n5-2012 > RIAF-V5N5-2012-6

Salcedo, I., \& Romero, J. J. (2006). Cultura Organizacional y Gestón de la Calidad en una Empresa del Estado venezolano. Revista Venezolana de Gerencia, 11(33).

Senplades. (noviembre de 2015). Secretaría Técnica Planifica Ecuador. Recuperado de Agenda Zonal 8: http://www.planificacion.gob.ec/wpcontent/uploads/downloads/2015/11/Agenda-zona-8.pdf

Sesento, L. (2012). Modelo Sistémico basado en competencias para instituciones educativas públicas. Centro de Investigación y desarrollo del Estado de Michoacán, Morelia (Tesis de doctorado). Recuperado de Eumed: http://www.eumed.net/tesis-doctorales/2012/lsg/concepto_modelo.html

Thomás, M., \& Ion, G. (2008). Contrastando dos modelos de análisis de la cultura organizativa de la universidad. Revista Bordón, 60 (3), 131-147. Recuperado de https://dialnet.unirioja.es

Thomás, M., \& Rodríguez, D. (marzo de 2009). Conocer la Cultura de la Universidad Contemporánea: CICOU. Revista Iberoamericana de Educación, Vol. 49, Núm.1. Recuperado de https://rieoei.org/RIE/article/view/2115

Times Higher Education. (enero de 2017). The Times Higher Education Young University Rankings list the world's best universities that are aged 50 years or under. Recuperado de https://www.timeshighereducation.com/world-universityrankings/young-university-rankings-2017-growing-concern-sprouts-new-branches

ISSN: 1390-9320, Edición Especial, febrero 2020 
Tomás, M., Borrell, N., Castro, D., Freixas, M., Bernabé, D., \& Fuentes, M. (2009). La Cultura Innovadora de las Universidades. Barcelona: Octaedro.

Tuning América Latina. (19 de noviembre de 2012). Información y Análisis de América Latina. Recuperado de http://www.tuningal.org/es/competencias/geologia

UNESCO. (2011). Manual de Gestión para Directores de Instituciones Educativas. Perú: Lance Gráfico.

UNESCO. (2013). Situaciones educativas de América Latina y el Caribe: Hacia la educación de calidad para todos al 2015. Santiago de Chile, Chile: Imbunche.

United Nations Educational Scientificand Cultural Organization (UNESCO) (2011). UNESCO and Education "everyone has the right to education". Francia: UNESCO.

Velasco, R. (2015). Modelo de Cultura Organizacional y alineación estratégica para una maquiladora de exportación. México: Instituto Tecnológico de Sonora.

Vesga, J. J. (2013). Cultura organizacional y sistemas de gestión de la calidad: una relación clave en la gestión de las institucioes de educación superior. Revista Guillermo Ockham, 11 (2), 89-100. 